\title{
Introduction to theory/modeling methods in photosynthesis
}

\author{
Francesco Buda
}

Received: 3 July 2009/Accepted: 13 July 2009/Published online: 31 July 2009

(C) The Author(s) 2009. This article is published with open access at Springerlink.com

\begin{abstract}
Theory and molecular modeling play an increasingly important role in complementing the experimental findings and supporting the interpretation of the data. Owing to the increase in computational power combined with the development of more efficient methods, computer simulations and modeling have emerged as primary ingredients of modern scientific inquiry. Here, we introduce the methods that in our view bring the largest promises in photosynthesis research, indicate how they have already contributed, and can in the near future assume a significant role in this field. Particular emphasis is given to density functional theory and its combination with molecular dynamics simulations. We point out the need for a multi-scale approach in facing the challenging task of describing processes which cover several orders of magnitude both in the time scale and in the size of the systems of interest.
\end{abstract}

Keywords DFT · Molecular dynamics - QMMM · Multi-scale simulations · Free energy calculations

$\begin{array}{ll}\text { Abbreviations } \\ \text { MD } & \text { Molecular dynamics } \\ \text { PES } & \text { Potential energy surface } \\ \text { DFT } & \text { Density functional theory } \\ \text { CPMD } & \text { Car-Parrinello molecular dynamics } \\ \text { QM/MM } & \text { Quantum mechanics/molecular mechanics }\end{array}$

Special issue of Photosynthesis Research "Basics and Applications of Biophysical Techniques in Photosynthesis and Related Processes".

F. Buda $(\bowtie)$

Leiden Institute of Chemistry, Leiden University,

P.O. Box 9502, 2300 RA Leiden, The Netherlands

e-mail: f.buda@chem.leidenuniv.nl

\section{Introduction}

The modeling and theoretical description of the complex phenomena involved in photosynthesis constitutes a challenging task. Ideally, using the quantum-mechanical dynamical evolution of the system one would be able to properly describe the phenomena involved in photosynthesis. Of course this is in practice still only a dream, since, in spite of the considerable progress in computational power, this program can be carried out only for very small molecules, but is certainly out of reach for the biological systems of interest in the context of photosynthesis. Compromises need to be made, and a clever combination of different approaches with different level of approximations, as well as a proper use of experimental input, appears to be the best strategy so far.

For the sake of clarity, we can distinguish between phenomenological semi-microscopic or macroscopic theories and microscopic models which take explicitly into account the atomistic details of the system.

\section{Phenomenological theories}

In phenomenological semi-microscopic or macroscopic approaches, the system is described by an effective Hamiltonian containing several parameters. For example, in theory of exciton coupling and excitation energy transfer in pigment-protein complexes (see e.g., Renger and Holzwarth 2008; Renger 2009 in this issue) the effective Hamiltonian contains the local transition energies of the pigments, optical transition dipole moments, and the excitonic couplings. These quantities need to be either derived from experimental data or, if these are not available, estimated by using a quantum-mechanical microscopic approach taking into 
account the specific structural details of the photosynthetic system of interest. Therefore, the two level of theoretical description mentioned above are actually interconnected.

\section{First-principles quantum-mechanical approaches (DFT, TD-DFT)}

The microscopic calculation of these parameters by the first-principles quantum-mechanical approach is by itself a difficult task because one needs to take into account the complex pigment-pigment and pigment-protein interactions. Accurate highly correlated wavefunction-based methods such as coupled cluster or the complete-activespace self-consistent-field (CASSCF) approach (see e.g., Cramer 2002) are computationally very expensive and can hardly deal with the large molecular models of interest in this context. Therefore, the quantum chemical method that is most widely used in applications related to biological systems or large molecular complexes is density functional theory (DFT) (see e.g., Dreizler and Gross 1990). The central quantity in DFT is the electron density, which depends only on three spatial coordinates. This constitutes an enormous simplification when compared to the manyelectron wavefunction, which depends on all electronic coordinates and whose complexity thus increases with the size of the system. The approximations in DFT are contained in the exchange-correlation functional, and the development of more accurate functional is a topic of current research (Gruning et al. 2004). DFT is a valuable tool to complement experimental investigations and even to predict, with a reasonable accuracy, many molecular properties such as geometries, reaction mechanisms, and spectroscopic properties (Wawrzyniak et al. 2008; Alia et al. 2009; Ganapathy et al. 2009a, b). An account on DFT and its applications to photosynthesis is presented in this issue by Orio et al. With the current computational power it has become feasible to treat systems containing several hundred of atoms and with accuracies comparable to more expensive wavefunction-based correlated methods. However, the intrinsically single-determinant nature of DFT poses some problems in the treatment of open-shell systems and particularly of multinuclear transition metal complexes, such as those involved in the catalytic water oxidation reactions (Rossmeisl et al. 2005; Siegbahn 2008; Lubitz et al. 2008; Herrmann et al. 2009).

DFT within the Hohenberg-Kohn formulation (Hohenberg and Kohn 1964) is designed for the electronic groundstate. In photosynthesis research it is desirable to have a theory that can describe both the optical properties and photo-induced processes. An accurate description of the electronic excited states is an extremely challenging problem in modern quantum chemistry (see e.g., Filippi et al. 2009). A generalization of DFT in the case of a timedependent external field has been formulated by Runge and Gross (1984). Within time-dependent DFT (TD-DFT) it is possible to compute properties like polarizabilities and excitation energies through a linear density response of the system. In spite of some known shortcomings of TD-DFT, such as a poor description of excited states with strong charge transfer character, this approach can be applied to large molecular complexes and provides a useful tool to interpret and complement experimental optical data. As an example, a recent TD-DFT study by Neugebauer (2008) has addressed the issue of the environmental effects on the excitation energies and photophysical properties of LH2 complexes (see also Orio et al. in this issue).

\section{Molecular dynamics}

Usually electronic structure calculations are performed on a fixed nuclear configuration (geometrical structure) within the Born-Oppenheimer approximation (see e.g., Atkins and Friedman 2005). By using the forces evaluated for that particular geometry, it is possible to find stationary states, minima, and saddle points, on the potential energy surface (PES). In general however, it would be desirable to include explicitly dynamical effects due to the nuclear motion at finite temperature and to obtain free energy surfaces along a specific reaction coordinate. This aim can be achieved by Molecular Dynamics (MD) simulations that represent a powerful tool to treat explicitly the atomic motion of a pigment-protein complex at realistic thermodynamic conditions and including solvent effects (Frenkel and Smit 1996). In this approach, the Newtonian equations of motion are solved numerically by evolving in time the positions and velocities of each particle by a very small time interval $\Delta t$ at each MD step. Typical values of the time step $\Delta t$ are of the order of $1 \mathrm{fs}$.

The PES, which is used to derive the atomic forces, is usually written in a simple functional form containing bonded terms, such as stretching, bending, and torsional energy, and non-bonded terms, most importantly electrostatic and van der Waals interactions. All these contributions to the total energy contain a number of empirical parameters that need to be predefined and that characterize a particular force field. Some of the most commonly used force fields for biomolecules are the AMBER and CHARMM force fields. MD simulations based on empirical force fields are widely used to study structure-function relationship in proteins with known crystal structures (see, e.g., Warshel 1991; Kosztin and Schulten 2008). This numerical technique has been applied to study the reorganization energy of the initial electron-transfer step in photosynthetic bacterial reaction centers (BRC) (Parson 
et al. 1998; Parson and Warshel 2008). The MD trajectories can be also used in combination with quantum chemical methods for predicting and characterizing charge transfer processes and optical properties (Damjanovic et al. 2002). For a review on successful applications and limitations of MD simulations for biochemical reactions, we refer to Warshel and Parson 2001. The use of an empirical force field for the PES allows for the dynamical simulation of rather large systems up to a million atoms and to explore time scales of the order of $100 \mathrm{~ns}$ (Klein and Shinoda 2008). Moreover atomistic simulations can be used to estimate parameters needed in so-called coarse-grain models or macroscopic theory.

\section{Ab initio MD}

One crucial limitation of MD simulations lies in the use of a predefined PES which is based on the knowledge of the molecular structure and bonding pattern. This assumption implies that processes, such as chemical bond breaking and formation, which may occur during the dynamical evolution of the system, cannot be described in this context. Moreover, the derivation of appropriate force fields for transition metal complexes such as the one involved in the catalytic water oxidation reaction in photosystem II is a very challenging task. It is clear that a proper quantum-mechanical (QM) description of the PES is needed if one wants to describe the chemical reactions relevant to photosynthesis. This can be done within the Born-Oppenheimer approximation by solving the electronic Schrödinger equation on the $f l y$, i.e., for each nuclear configuration explored along the MD trajectory. This scheme can be defined by the coupled equations:

$M_{\mathrm{I}} \frac{d^{2} R_{\mathrm{I}}}{d t^{2}}=-\nabla_{\mathrm{I}}\left\langle\Psi_{0}\left|H_{\mathrm{e}}\right| \Psi_{0}\right\rangle$

$H_{\mathrm{e}} \Psi_{0}=E_{0} \Psi_{0}$

Equation 1 is the Newton's second law of motion for the nucleus I with mass $M_{\mathrm{I}}$ and position $R_{\mathrm{I}}$. The force that appears on the right-hand side of Eq. 1 is obtained by calculating the gradient $\left(\nabla_{\mathrm{I}}\right)$ of the total energy with respect to the nuclear position $R_{\mathrm{I}}$. The total energy is in turn obtained as the expectation value of the electronic Hamiltonian $H_{\mathrm{e}}$, which depends parametrically on the nuclear positions $R_{\mathrm{I}}$. The Hamiltonian $H_{\mathrm{e}}$ includes also the nuclei-nuclei repulsion term. Equation 2 is the electronic Schrödinger equation, where $\Psi_{0}$ and $E_{0}$ are the ground-state electronic wavefunction and energy, respectively. The first-principles molecular dynamics approach derived from these equations, implicitly assumes (i) the Born-Oppenheimer approximation that allows us to separate the electronic and the nuclear dynamics, (ii) the classical approximation for the nuclear motion. An efficient scheme to solve Eqs. 1 and 2 has been developed in 1985 in what is now usually called the CarParrinello molecular dynamics method (CPMD) (Car and Parrinello 1985). This approach is based on an efficient algorithm for solving the Schrödinger equation, and it takes advantage of the continuity of the dynamical trajectories in order to compute with a minimum computational effort the new electronic ground-state after each atomic step in the trajectory. In the CPMD method, DFT is generally used for computing the electronic ground-state energy. Though DFT is not necessarily the only possible choice (the same formalism can in fact be developed using, e.g., the HartreeFock approximation for the electrons), it is the most convenient one for the advantageous scaling property and accuracy of DFT. In the CPMD method therefore no empirical parameter is required for the PES and the only input required in the simulation is essentially the atomic number of the atomic constituents.

The use of the first-principles PES has several advantages over the empirical potentials: (i) the PES is fully transferable, i.e., it can be used for a cluster as well as for an extended system in different condensed phases without the need for re-parameterization; (ii) chemical reactions can be simulated since bond breaking and forming are allowed by the rearrangement of the electronic density along the trajectory; (iii) increased predictive power of the simulation. Of course the price of using the first-principles PES is a much larger computational cost of the simulation. At present, CPMD simulations can handle systems consisting of a few hundred atoms, and can follow the trajectory for a time of the order of $10 \mathrm{ps}$.

\section{QM/MM methods}

The processes of interest in natural photosynthesis are characterized by very large pigment-protein complexes containing many thousands atoms and span several orders of magnitude in the time scale (from ps to $\mathrm{ms}$ or more). Therefore, in spite of the considerable progress done in first-principles calculations and in particular in DFT-based methods, we still need to develop novel multiscale methods combining different approaches with different accuracies and computational cost, which may be able to deal with these challenging questions.

A first step in this direction is realized by hybrid quantum mechanics-molecular mechanics (QM/MM) approaches where a quantum mechanics calculation is embedded in a classical molecular mechanics model of the environment. In the QM/MM scheme, we can incorporate in the simulation the environmental effects at an atomistic level, such as mechanical constraints, electrostatic 
perturbations, and dielectric screening. The idea of a QM/ MM scheme is not new and the first published example appeared already more than thirty years ago (Warshel and Levitt 1976). However, in the last few years this subject has developed very rapidly and different implementations of QM/MM approaches have appeared in the literature. For recent reviews, see, e.g., Sherwood (2000) and Lin and Truhlar (2007). The first step in a QM-MM simulation is to divide the system in two subsystems: One "inner" (usually small) region which is treated with quantum mechanics $(\mathrm{QM})$ and an "outer" region which is treated with molecular mechanics (MM). The basis for this separation is that the region of space where the QM approach is needed is usually limited to a relatively small region where the electronic structure changes significantly (bond-making and bond-breaking processes) during the simulation. For example, we can consider an enzyme, whose active site is the region where the chemical reaction takes place and therefore must be treated with $\mathrm{QM}$. The remainder of the enzyme is important for maintaining its proper structure, folding, etc., but can be treated with a classical force field approach (MM). In the context of photosynthesis an interesting $\mathrm{QM} / \mathrm{MM}$ application has recently appeared describing the catalytic cycle of the oxygen evolving complex in photosystem II (Sproviero et al. 2008, 2009, in this issue).

\section{Concluding remarks and outlook}

The development of embedding schemes, such as QM/ $\mathrm{MM}$, is particularly promising for the description of the catalytic reactions both in natural and artificial photosynthesis. The frozen density embedding method is an example of a recent $\mathrm{QM} / \mathrm{QM}$ embedding scheme which appears very interesting in this context (Neugebauer 2008). Ab initio MD and QM/MM simulations can be generalized to electronic excited states provided the excited-state PES can be predicted with reasonable accuracy. Methods for excited-state PES such as TD-DFT are quite promising in this respect, but more applications and accuracy assessment are needed. It can also be expected in the near future that new exchange-correlation functionals will be developed to improve the description of excited states and magnetic effects in multi-nuclear transition metal complexes (Herrmann et al. 2009). Another sector that has recently witnessed a considerable progress is the development of methods for the prediction of free energy surfaces, such as the metadynamics approach (Laio and Parrinello 2002). In conclusion, available theoretical and computational approaches provide a crucial tool complementary to experimental data and are able to predict molecular properties and reaction pathways with fair accuracy, opening the possibility of in-silico design of novel catalysts. Theoretical and methodological developments are needed especially in the direction of multi-scale approaches possibly combining atomistic with mesoscopic scale simulations.

Open Access This article is distributed under the terms of the Creative Commons Attribution Noncommercial License which permits any noncommercial use, distribution, and reproduction in any medium, provided the original author(s) and source are credited.

\section{References}

Alia A, Wawrzyniak PK, Janssen G, Buda F, Matysik J, de Groot HJM (2009) Differential charge polarization of axial histidines in bacterial reaction centres balances the asymmetry of the special pair. J Am Chem Soc 131:9626-9627. doi: 10.1021/ ja9028507

Atkins PW, Friedman RS (2005) Molecular quantum mechanics. Oxford University Press, New York

Car R, Parrinello M (1985) Unified approach for molecular dynamics and density-functional theory. Phys Rev Lett 55:2471-2474

Cramer CJ (2002) Essentials of computational chemistry-Theories and models. Wiley, Chichester

Damjanovic A, Kosztin I, Kleinekathofer U, Schulten K (2002) Excitons in a photosynthetic light-harvesting system: a combined molecular dynamics, quantum chemistry, and polaron model study. Phys Rev E 65:031919-1-031919-24

Dreizler RM, Gross EKV (1990) Density functional theory. Springer, Berlin

Filippi C, Zaccheddu M, Buda F (2009) The absorption spectrum of the green fluorescent protein chromophore: a difficult case for ab initio methods? J Chem Theory Comput. doi:10.1021/ ct900227j

Frenkel D, Smit B (1996) Understanding molecular simulationFrom algorithms to applications. Academic Press, San Diego

Ganapathy S, Oostergetel G, Wawrzyniak PK, Reus M, Gomez A, Chew M, Buda F, Boekema E, Holzwarth A, Bryant D, de Groot HJM (2009a) Alternating syn-anti bacteriochlorophylls form concentric helical nanotubes in chlorosomes. PNAS 106:85258530

Ganapathy S, Sengupta S, Wawrzyniak PK, Huber V, Buda F, Baumeister U, Würthner F, de Groot HJM (2009b) Zinc chlorins for artificial light-harvesting self-assemble into antiparallel stacks forming a microcrystalline solid-state material. PNAS 106:11472-11477

Gruning M, Gritsenko OV, Baerends EJ (2004) Improved description of chemical barriers with generalized gradient approximations (GGAs) and meta-GGAs. J Phys Chem A 108:4459-4469

Herrmann C, Podewitz M, Reiher M (2009) Restrained optimization of broken-symmetry determinants. Int $\mathrm{J}$ Quantum Chem 109:2430-2446

Hohenberg P, Kohn W (1964) Inhomogeneous electron gas. Phys Rev B 136:864-871

Klein ML, Shinoda W (2008) Large-scale molecular dynamics simulations of self-assembling systems. Science 321:798-800

Kosztin I, Schulten K (2008) Molecular dynamics methods for bioelectronic systems in photosynthesis. In: Aartsma TJ, Matysik $\mathrm{J}$ (eds) Biophysical techniques in photosynthesis, vol 2, Series advances in photosynthesis and respiration, vol 26. Springer, Dordrecht, pp 445-464

Laio A, Parrinello M (2002) Escaping free-energy minima. PNAS 99:12562-12566 
Lin H, Truhlar DG (2007) QM/MM: what have we learned, where are we, and where do we go from here? Theory Chem Acc 117:185199

Lubitz W, Reijerse EJ, Messinger J (2008) Solar water-splitting into $\mathrm{H}_{2}$ and $\mathrm{O}_{2}$ : design principles of photosystem II and hydrogenases. Energy Environ Sci 1:15-31

Neugebauer J (2008) Photophysical properties of natural lightharvesting complexes studied by subsystem density functional theory. J Phys Chem B 112:2207-2217

Parson WW, Warshel A (2008) Calculations of electrostatic energies in proteins using microscopic, semimicroscopic and macroscopic models and free-energy perturbation approaches. In: Aartsma TJ, Matysik J (eds) Biophysical techniques in photosynthesis, vol 2, Series advances in photosynthesis and respiration, vol 26. Springer, Dordrecht, pp 401-420

Parson WW, Chu ZT, Warshel A (1998) Reorganization energy of the initial electron-transfer step in photosynthetic bacterial reaction centers. Biophys J 74:182-191

Renger T, Holzwarth AR (2008) Theory of excitation energy transfer and optical spectra of photosynthetic systems. In: Aartsma TJ, Matysik J (eds) Biophysical techniques in photosynthesis, vol 2, Series advances in photosynthesis and respiration, vol 26. Springer, Dordrecht, pp 421-443

Rossmeisl J, Logadottir A, Nørskov JK (2005) Electrolysis of water on (oxidized) metal surfaces. Chem Phys 319:178-184
Runge E, Gross EKU (1984) Density-functional theory for timedependent systems. Phys Rev Lett 52:997-1000

Sherwood P (2000) Hybrid quantum mechanics/molecular mechanics approaches. In: Grotendorst $\mathrm{J}$ (ed) Modern methods and algorithms of quantum chemistry, vol 1. NIC Series, Jülich, pp 257-277

Siegbahn PEM (2008) A structure-consistent mechanism for dioxygen formation in photosystem II. Chem Eur J 14:8290-8302

Sproviero EM, Gascon JA, McEvoy JP, Brudvig GW, Batista VS (2008) QM/MM study of the catalytic cycle of water splitting in photosystem II. J Am Chem Soc 130:3428-3442

Warshel A (1991) Computer modeling of chemical reactions in enzymes and solutions. Wiley, New York

Warshel A, Levitt M (1976) Theoretical studies of enzymic reactions: dielectric, electrostatic and steric stabilisation of the carbonium ion in the reaction of lysozyme. J Mol Biol 103:227-249

Warshel A, Parson WW (2001) Dynamics of biochemical and biophysical reactions: insight from computer simulations. Q Rev Biophys 34:563-679

Wawrzyniak PK, Alia A, Schaap RG, Heemskerk MM, de Groot HJM, Buda F (2008) Protein-induced geometric constraints and charge transfer in bacteriochlorophyll-histidine complexes in LH2. Phys Chem Chem Phys 10:6971-6978 